DOI: https://doi.org/10.24297/jam.v18i.8744

\title{
Recent Modification of Decomposition Method for Solving Nonlinear Partial Differential Equations
}

\author{
Zainab Hadi Kareem and Luma Naji Mohammed Tawfiq ${ }^{\star}$ \\ College of Education for Pure Science ibn Al-Haitham, University of Baghdad, Baghdad, Iraq \\ *luma.n.m@ihcoedu.uobaghdad.edu.iq
}

\begin{abstract}
In this paper, efficient modification of Adomain decomposition method is proposed to solve nonlinear partial differential equations. Yields solution in rapid convergent series from easily computable terms to get exact solution, and yields in few iterations we get exact solution. Moreover, this modification does not require any linearization, discretization, or perturbations and therefore reduces the computations. Two illustration examples are introduced and illustrate the procedure of modification is simple yet highly accurate and rapidly converge to exact solution compares with the ADM or other modifications. The methodology presented here is useful for strongly nonlinear problems.
\end{abstract}

Keywords: Pdes, ADM, Modified Decomposition Method, Convergence Analysis.

\section{Introduction}

In the last three decades, the Adomian Decomposition Method (ADM) has confirmed successful in getting analytical solution of non-linear differential equations by obtained solution in terms of convergent power series [1]. This method does not require discretization of the variables or domain $[2,3]$. The theoretical analysis of convergence for the series solution of ADM has been studied by Adomian [4], Cherrault [5], Cherrault et al. [6], and Chrysos et al. [7].

In recent years, there has been development in the application of ADM in solving partial differential equations (PDEs) with variable coefficients. For example, Wazwaz and Gorguis [8] also used the ADM solved linear heatlike and wave-like equations with variable coefficients. Soufyane and Boulmaf [9] applied ADM to get analytical solutions to the non-linear parabolic equation with variable physical parameters in time and space. Achouri and Omrani [10], applied the ADM to get numerical solutions for the damped generalized regularized long-wave equation (DGRLW) with variable coefficients, and Tawfiq et al. [11-14] used this method for solving different model equations.

The aim of this paper is to get the exact analytical solution of nonlinear PDEs by using new efficient modification of ADM.

\section{Suggested Modification of ADM}

The procedure of the suggested modification for decomposition method (MADM) is introduced. Firstly writes the equation of nonlinear PDEs as:

$L_{t} u+R u(x, t)+N u(x, t)=0$

With the IC: $u(x, 0)=f(x)$

Where $x \in R, L$ : is a lower linear differential operator $\left(L_{t}=\frac{\partial}{\partial t}\right) ; R$ : is a is a remainder linear operator, which has partial derivatives with respect to $x$. 
$N$ :is a nonlinear operator and $L_{t}^{-1}=\int_{0}^{t}() d$.

Take $\square_{\square}^{-1}$ for the equation (1), we have

$u(x, t)=f(x)-L_{t}^{-1}[R u(x, t)]-L_{t}^{-1}[N u(x, t)]$

The idea of modification is represented here by decomposed unknown function $u(x, t)$ by infinite series as:

$u(x, t)=\sum_{m=0}^{\infty} a_{m}(x) t^{m}$

Also, the nonlinear term $N u(x, t)$ can be represented by an infinite series of polynomials as follows:

$N u(x, t)=\sum_{m=0}^{\infty} A_{m}(x) t^{m}=A_{0}+A_{1} t+A_{2} t^{2}+\cdots$

Then substitute equations (4) and (5) in (3) to obtain:

$\sum_{m=0}^{\infty} a_{m}(x) t^{m}=f(x)-L_{t}^{-1} R\left(\sum_{m=0}^{\infty} a_{m}(x) t^{m}\right)-L_{t}^{-1}\left(\sum_{m=0}^{\infty} A_{m}(x) t^{m}\right)$

Now, integration the right side, we have

$\sum_{m=0}^{\infty} a_{m}(x) t^{m}=f(x)-R\left(\sum_{m=0}^{\infty} a_{m}(x) \frac{t^{m+1}}{m+1}\right)-\left(\sum_{m=0}^{\infty} A_{m}(x) \frac{t^{m+1}}{m+1}\right)$

let $\mathrm{m}=\mathrm{m}-1$ on the right side of the above system, to get:

$\sum_{m=0}^{\infty} a_{m}(x) t^{m}=f(x)-R\left(\sum_{m=0}^{\infty} a_{m-1}(x) \frac{t^{m}}{m}\right)-\left(\sum_{m=0}^{\infty} A_{m-1}(x) \frac{t^{m}}{m}\right)$

let $a_{0}(x)=f(x)$

$a_{m}(x)=\frac{1}{m}\left[-R\left(a_{m-1}(x)\right)-A_{m-1}(x)\right]$

From equation (7) $\mathrm{a}_{\mathrm{m}}$ can be determining then substitute it in equation (4) to get the solution $u(x, t)$.

\section{Illustrative Applications}

Here, the suggested modification will be used to solve the nonlinear PDE with initial condition as the following:

\section{Example 1}

Consider the following $3^{\text {rd }}$ order nonlinear PDEs:

$u_{t}+6 u^{2} u_{x}+u_{x x x}=0, \quad x \epsilon R$,

with IC: $\boldsymbol{u}(\boldsymbol{x}, 0)=\boldsymbol{k} \operatorname{sech}(\boldsymbol{k} x)$

To solve the equation by suggested modification, first we take $=\frac{\partial}{\partial t}$, so 


$$
L_{t}^{-1}=\int_{0}^{t}(.) d t
$$

Take $L_{t}^{-1}$ to both side of equation, we have

$$
\begin{aligned}
& u(x, t)=u(x, 0)+L_{t}^{-1}\left[-6 u^{2} u_{x}-u_{x x x}\right] \\
& u(x, t)=k \operatorname{sech}(k x)-6 L_{t}^{-1}\left[u^{2} u_{x}\right]-\frac{\partial^{3}}{\partial x^{3}} L_{t}^{-1}[u] \\
& \text { let } u(x, t)=\sum_{m=0}^{\infty} a_{m}(x) t^{m} \\
& \sum_{m=0}^{\infty} a_{m}(x) t^{m}=k \operatorname{sech}(k x)-6\left(\sum_{m=0}^{\infty} A_{m}(x) \frac{t^{m+1}}{m+1}\right)-\frac{\partial^{3}}{\partial x^{3}}\left(\sum_{m=0}^{\infty} a_{m}(x) \frac{t^{m+1}}{m+1}\right)
\end{aligned}
$$

where the nonlinear term defined as:

$\boldsymbol{u}^{2} \boldsymbol{u}_{\boldsymbol{x}}=\sum_{m=0}^{\infty} A_{m}(x) t^{m}=A_{0}+A_{1} t+A_{2} t^{2}+\cdots$

let $m=m-1$ in the right side of the above equation, we have

$$
\begin{aligned}
& \sum_{m=0}^{\infty} a_{m}(x) t^{m}=k \operatorname{sech}(k x)-6\left(\sum_{m=0}^{\infty} A_{m-1}(x) \frac{t^{m}}{m}\right)-\frac{\partial^{3}}{\partial x^{3}}\left(\sum_{m=0}^{\infty} a_{m-1}(x) \frac{t^{m}}{m}\right) \\
& a_{0}(x)=k \operatorname{sech}(k x) \\
& a_{m}(x)=\frac{1}{m}\left[-6\left(A_{m-1}(x)\right)-\frac{\partial^{3}}{\partial x^{3}}\left(a_{m-1}(x)\right)\right] \\
& a_{1}(x)=\frac{1}{1}\left[-6\left(A_{0}\right)-\frac{\partial^{3}}{\partial x^{3}}\left(a_{0}(x)\right)\right] \\
& a_{1}(x)=\left[-6\left(-k^{4} \tanh (k x) \operatorname{sech}^{3}(k x)\right)-\frac{\partial^{3}}{\partial x^{3}}(k \operatorname{sech}(k x))\right] \\
& a_{1}(x)=\left[6 k^{4} \tanh (k x) \operatorname{sech}^{3}(k x)-5 k^{4} \tanh (k x) \operatorname{sech}^{3}(k x)+k^{4} \tanh ^{3}(k x) \operatorname{sech}(k x)\right] \\
& a_{1}(x)=\left[k^{4} \tanh (k x) \operatorname{sech}^{3}(k x)+k^{4} \tanh ^{3}(k x) \operatorname{sech}(k x)\right] \\
& a_{1}(x)=k^{4} \tanh (k x) \operatorname{sech}(k x) \\
& a_{2}(x)=\frac{1}{2}\left[-6\left(A_{1}\right)-\frac{\partial^{3}}{\partial x^{3}}\left(a_{1}(x)\right)\right] \\
& a_{2}(x)=\frac{1}{2}\left[-6\left(-3 k^{7} \tanh ^{2}(k x) \operatorname{sech}^{3}(k x) t+k^{7} \operatorname{sech}^{5}(k x)\right)-\frac{\partial^{3}}{\partial x^{3}}(k \operatorname{sech}(k x))\right] \\
& a_{2}(x)=\frac{1}{2}\left[18 k^{7} \tanh ^{2}(k x) \operatorname{sech}^{3}(k x)-6 k^{7} \operatorname{sech}^{5}(k x) t+5 k^{7} \operatorname{sech}^{5}(k x)-18 k^{7} \tanh ^{2}(k x) \operatorname{sech}^{3}(k x)+\right. \\
& k^{7} \tanh ^{4}(k x) \operatorname{sech}(k x) \text { ] }
\end{aligned}
$$




$$
\begin{aligned}
& a_{2}(x)=\frac{1}{2}\left[-k^{7} \operatorname{sech}^{5}(k x)+k^{7} \tanh ^{4}(k x) \operatorname{sech}(k x)\right] \\
& a_{2}(x)=\frac{1}{2}\left[k^{7} \operatorname{sech}(k x)\left(\tanh ^{4}(k x)-\operatorname{sech}^{4}(k x)\right)\right] \\
& a_{2}(x)=\frac{1}{2}\left[k^{7} \operatorname{sech}(k x)\left(\tanh ^{2}(k x)-\operatorname{sech}^{2}(k x)\right)\right] \\
& a_{2}(x)=\frac{1}{2}\left[-\frac{k^{7}}{2} \operatorname{sech}^{3}(k x)(3-\cosh (2 k x))\right] \\
& a_{2}(x)=-\frac{k^{7}}{4} \operatorname{sech}^{3}(k x)(3-\cosh (2 k x))
\end{aligned}
$$

Therefore,

$u(x, t)=\sum_{m=0}^{\infty} a_{m}(x) t^{m}=a_{0}+a_{1} t+a_{2} t^{2}+\cdots$

$u(x, t)=k \operatorname{sech}(k x)+k^{4} \tanh (k x) \operatorname{sech}(k x)-\frac{k^{7}}{4} \operatorname{sech}^{3}(k x)(3-\cosh (2 k x)) t^{2}+\cdots$

It is clear that the series is closed to

$u(x, t)=k \operatorname{sech}\left(k\left(x-k^{2} t\right)\right)$

This is exact solution.

\section{Example 2}

Consider the following $3^{\text {rd }}$ order nonlinear PDEs:

$u_{t}-6 u u_{x}+u_{x x x}=0, \quad x \in R$,

with IC: $u(x, 0)=\frac{-k^{2}}{2} \operatorname{Sech}^{2}\left(\frac{k}{2} x\right)$

\section{Solution}

To solve the equation by suggested modification, first we take $=\frac{\partial}{\partial t}$, so

$$
L_{t}^{-1}=\int_{0}^{t}(.) d t
$$

Take $L_{t}^{-1}$ to both side of an above equation, we have

$$
\begin{aligned}
& u(x, t)=u(x, 0)+L_{t}^{-1}\left[6 u u_{x}-u_{x x x}\right] \\
& u(x, t)=\frac{-k^{2}}{2} \operatorname{Sech}^{2}\left(\frac{k}{2} x\right)+6 L_{t}^{-1}\left[u u_{x}\right]-\frac{\partial^{3}}{\partial x^{3}} L_{t}^{-1}[u] \\
& \text { let } u(x, t)=\sum_{m=0}^{\infty} a_{m}(x) t^{m}
\end{aligned}
$$


$\sum_{m=0}^{\infty} a_{m}(x) t^{m}=\frac{-k^{2}}{2} \operatorname{Sech}^{2}\left(\frac{k}{2} x\right)+6\left(\sum_{m=0}^{\infty} A_{m}(x) \frac{t^{m+1}}{m+1}\right)-\frac{\partial^{3}}{\partial x^{3}}\left(\sum_{m=0}^{\infty} a_{m}(x) \frac{t^{m+1}}{m+1}\right)$

where the nonlinear term defined as:

$u u_{x}=\sum_{m=0}^{\infty} A_{m}(x) t^{m}=A_{0}+A_{1} t+A_{2} t^{2}+\cdots$

let $m=m-1$ on the right side of the above equation, we have

$\sum_{m=0}^{\infty} a_{m}(x) t^{m}=\frac{-k^{2}}{2} \operatorname{Sech}^{2}\left(\frac{k}{2} x\right)+6\left(\sum_{m=0}^{\infty} A_{m-1}(x) \frac{t^{m}}{m}\right)-\frac{\partial^{3}}{\partial x^{3}}\left(\sum_{m=0}^{\infty} a_{m-1}(x) \frac{t^{m}}{m}\right)$

$a_{0}(x)=\frac{-k^{2}}{2} \operatorname{Sech}^{2}\left(\frac{k}{2} x\right)$

$a_{m}(x)=\frac{1}{m}\left[6\left(A_{m-1}(x)\right)-\frac{\partial^{3}}{\partial x^{3}}\left(a_{m-1}(x)\right)\right]$

$a_{1}(x)=\frac{1}{1}\left[6\left(A_{0}\right)-\frac{\partial^{3}}{\partial x^{3}}\left(a_{0}(x)\right)\right]$

$a_{1}(x)=\left[6\left(-\frac{k^{5}}{4} \tanh \left(\frac{k}{2} x\right) \operatorname{Sech}^{4}\left(\frac{k}{2} x\right)\right)-\frac{\partial^{3}}{\partial x^{3}}\left(\frac{-k^{2}}{2} \operatorname{Sech}^{2}\left(\frac{k}{2} x\right)\right)\right]$

$a_{1}(x)=\left[-\frac{3 k^{5}}{2} \tanh \left(\frac{k}{2} x\right) \operatorname{Sech}^{4}\left(\frac{k}{2} x\right)-\frac{k^{5}}{2} \tanh ^{3}\left(\frac{k}{2} x\right) \operatorname{Sech}^{2}\left(\frac{k}{2} x\right)+k^{5} \tanh \left(\frac{k}{2} x\right) \operatorname{Sech}^{4}\left(\frac{k}{2} x\right)\right]$

$a_{1}(x)=\left[-\frac{k^{5}}{2} \tanh \left(\frac{k}{2} x\right) \operatorname{Sech}^{4}\left(\frac{k}{2} x\right)-\frac{k^{5}}{2} \tanh ^{3}\left(\frac{k}{2} x\right) \operatorname{Sech}^{2}\left(\frac{k}{2} x\right)\right]$

$a_{1}(x)=\left[-\frac{k^{5}}{2} \tanh \left(\frac{k}{2} x\right) \operatorname{Sech}^{2}\left(\frac{k}{2} x\right)\left(\operatorname{Sech}^{2}\left(\frac{k}{2} x\right)+\tanh ^{2}\left(\frac{k}{2} x\right)\right)\right]$

$a_{1}(x)=-\frac{k^{5}}{2} \tanh \left(\frac{k}{2} x\right) \operatorname{Sech}^{2}\left(\frac{k}{2} x\right)$

$a_{2}(x)=\frac{1}{2}\left[6\left(A_{1}\right)-\frac{\partial^{3}}{\partial x^{3}}\left(a_{1}(x)\right)\right]$

$a_{2}(x)=\frac{1}{2}\left[6\left(-\frac{2 k^{8}}{4} \tanh ^{2}\left(\frac{k}{2} x\right) \operatorname{Sech}^{4}\left(\frac{k}{2} x\right)+\frac{k^{8}}{8} \operatorname{Sech}^{6}\left(\frac{k}{2} x\right)\right)-\frac{\partial^{3}}{\partial x^{3}}\left(-\frac{k^{5}}{2} \tanh \left(\frac{k}{2} x\right) \operatorname{Sech}^{2}\left(\frac{k}{2} x\right)\right)\right]$

$a_{2}(x)=\frac{1}{2}\left[-3 k^{8} \tanh ^{2}\left(\frac{k}{2} x\right) \operatorname{Sech}^{4}\left(\frac{k}{2} x\right)+\frac{3 k^{8}}{4} \operatorname{Sech}^{6}\left(\frac{k}{2} x\right) t-\frac{k^{8}}{2} \operatorname{Sech}^{6}\left(\frac{k}{2} x\right)\right.$

$$
\left.+\frac{11 k^{8}}{4} \tanh ^{2}\left(\frac{k}{2} x\right) \operatorname{Sech}^{4}\left(\frac{k}{2} x\right)-\frac{k^{8}}{2} \tanh ^{4}\left(\frac{k}{2} x\right) \operatorname{Sech}^{2}\left(\frac{k}{2} x\right)\right]
$$

$a_{2}(x)=\frac{1}{2}\left[-\frac{k^{8}}{4} \tanh ^{2}\left(\frac{k}{2} x\right) \operatorname{Sech}^{4}\left(\frac{k}{2} x\right)+\frac{k^{8}}{4} \operatorname{Sech}^{6}\left(\frac{k}{2} x\right)-\frac{k^{8}}{2} \tanh ^{4}\left(\frac{k}{2} x\right) \operatorname{Sech}^{2}\left(\frac{k}{2} x\right)\right]$ 
$a_{2}(x)=\frac{1}{2}\left[-\frac{k^{8}}{4} \tanh ^{2}\left(\frac{k}{2} x\right) \operatorname{Sech}^{2}\left(\frac{k}{2} x\right)\left(\operatorname{Sech}^{2}\left(\frac{k}{2} x\right)+2 \tanh ^{2}\left(\frac{k}{2} x\right)\right)+\frac{k^{8}}{4} \operatorname{Sech}^{6}\left(\frac{k}{2} x\right)\right]$

$a_{2}(x)=\frac{1}{2}\left[\frac{k^{8}}{4} \operatorname{Sech}^{4}\left(\frac{k}{2} x\right)(-\cosh (k x)+2)\right]$

$a_{2}(x)=\frac{k^{8}}{8} \operatorname{Sech}^{4}\left(\frac{k}{2} x\right)(2-\cosh (k x))$

Therefore,

$u(x, t)=\sum_{m=0}^{\infty} a_{m}(x) t^{m}=a_{0}+a_{1} t+a_{2} t^{2}+\cdots$

$u(x, t)=\frac{-k^{2}}{2} \operatorname{Sech}^{2}\left(\frac{k}{2} x\right)-\frac{k^{5}}{2} \tanh \left(\frac{k}{2} x\right) \operatorname{Sech}^{2}\left(\frac{k}{2} x\right) t+\frac{k^{8}}{8} \operatorname{Sech}^{4}\left(\frac{k}{2} x\right)(2-\cosh (k x)) t^{2}+\cdots$

It is clear that the series is closed to

$u(x, t)=\frac{-k^{2}}{2} \operatorname{Sech}^{2}\left(\frac{k}{2}\left(x-k^{2} t\right)\right)$

This is exact solution.

\section{The Convergence Analysis of Suggested Modification}

In this section the convergence of the MADM will be discuss. Now, consider the general form of equation as:

$$
\mathrm{u}-\mathrm{Nu}=\mathrm{f} \quad ; \mathrm{u} \in \mathrm{H}
$$

where $\mathrm{H}$ is the Hilbert space, $\mathrm{N}$ is the nonlinear operator $\mathrm{N}$ : $\mathrm{H} \rightarrow \mathrm{H}$ and $\mathrm{f}$ is also in $\mathrm{H}$. Substituting the decomposition series equations (5) and (9) in equation (10) to get:

$$
\sum_{n=0}^{\infty} u_{n}(x, t)-\sum_{n=0}^{\infty} A_{n}(x, t)=f
$$

So, the recursive terms are got by: $u_{0}=f$ and $u_{n+1}=A_{n}\left(u_{0}, u_{1}, \ldots, u_{n}\right)$

MADM suggest finding $B n=u_{1}+u_{2}+u_{3}+\ldots+u_{n}$ by using iterative scheme $B_{0}=0$

$B_{n+1}=N\left(B n+u_{0}\right)$, where

$$
\mathrm{N}\left(\mathrm{Bn}+\mathrm{u}_{0}\right)=\sum_{i=0}^{n} A_{i}
$$

If the limit exist in a Hilbert space

$$
\mathrm{B}=\lim _{n \rightarrow \infty} B_{n}
$$

then $B$ is a solution of the equation $B=N\left(u_{0}+B\right)$ in $H$.

\section{Theorem 1}

Let $\mathrm{N}$ be a nonlinear operator, $\mathrm{N}: \mathrm{H} \rightarrow \mathrm{H}$ where $\mathrm{H}$ is Hilbert space and $\mathrm{u}$ be the exact solution of equation (10). The decomposition series $\sum_{n=0}^{\infty} u_{n}(x, t)$ converges to $\mathrm{u}$ when 
Proof

$\exists \gamma<1 ;\left\|u_{n+1}\right\| \leq \gamma\left\|u_{n}\right\|, n \in z$

We need to prove the sequence $B_{n}=u_{1}+u_{2}+u_{3}+\ldots+u_{n}$, is a Cauchy sequence in the Hilbert space $H$.

$\left\|\mathrm{B}_{\mathrm{n}+1}-\mathrm{B}_{\mathrm{n}}\right\|=\left\|u_{n+1}\right\| \leq \gamma\left\|u_{n}\right\| \leq \gamma^{2}\left\|u_{n-1}\right\| \leq \cdots \leq \gamma^{n+1}\left\|u_{0}\right\|$

Now, we show $B_{n}$ is Cauchy sequence:

$$
\begin{aligned}
\left\|\mathrm{B}_{\mathrm{m}}-\mathrm{B}_{\mathrm{n}}\right\| & =\left\|\left(\mathrm{B}_{\mathrm{m}}-\mathrm{B}_{\mathrm{m}-1}\right)-\left(\mathrm{B}_{\mathrm{m}-1}-\mathrm{B}_{\mathrm{m}-2}\right)-\cdots-\left(\mathrm{B}_{\mathrm{n}+1}-\mathrm{B}_{\mathrm{n}}\right)\right\| \\
& \leq\left\|\mathrm{B}_{\mathrm{m}}-\mathrm{B}_{\mathrm{m}-1}\right\|+\left\|\mathrm{B}_{\mathrm{m}-1}-\mathrm{B}_{\mathrm{m}-2}\right\|+\left\|\mathrm{B}_{\mathrm{m}-2}-\mathrm{B}_{\mathrm{m}-3}\right\|+\cdots+\left\|\mathrm{B}_{\mathrm{n}+1}-\mathrm{B}_{\mathrm{n}}\right\| \\
& \leq \gamma^{m}\left\|u_{0}\right\|+\gamma^{m-1}\left\|u_{0}\right\|+\ldots+\gamma^{n+1}\left\|u_{0}\right\| \\
& =\left(\gamma^{m}+\gamma^{m-1}+\cdots+\gamma^{n+1}\right)\left\|u_{0}\right\| \\
& \leq\left(\gamma^{n+1}+\gamma^{n+2}+\cdots\right)\left\|u_{0}\right\|
\end{aligned}
$$

So, $\left\|\mathrm{B}_{\mathrm{m}}-\mathrm{B}_{\mathrm{n}}\right\|=\frac{\gamma^{n+1}}{1-\gamma}\left\|u_{0}\right\|$, for $\mathrm{n}, \mathrm{m} \in \mathrm{N} ; \mathrm{m} \geq \mathrm{n}$

Since $\gamma<1$, the sequence $\mathrm{B}_{\mathrm{n}}, \mathrm{n}=0, \ldots, \infty$ is a Cauchy sequence in the Hilbert space. Hence, $\lim _{n \rightarrow \infty} B_{n}=$ B.

so $B$ is the solution of equation (10).

\section{Conclusions}

In this research we consider the homogeneous nonlinear PDEs with variable coefficients and initial condition. To solve it, new efficient modification of ADM is applied. Then, it is seen that suggested modification has the same exact solution. In addition to their effectiveness and usefulness in solving nonlinear PDEs, we show that this modification is powerful tools in solving nonlinear PDEs with initial conditions. Compared to ADM, other modification, or other methods for solving nonlinear PDEs, there is no need for linearization of nonlinear terms (16-17), no need to calculate Adomian polynomial. Moreover, we can easily and rapidly attain the solution if we use MADM compared with ADM or other modifications as shown in solving illustrated examples.

\section{References}

1. AM Wazwaz. Partial Differential Equations and Solitary Waves Theory, $1^{\text {st }}$ ed. Beijing and Berlin, Springer; 2009, ISBN 978-3-642-00250-2, e-ISBN 978-3-642-00251-9.

2. LNM Tawfiq, KA Jasim and EO Abdulhmeed. Numerical Model for Estimation the Concentration of Heavy Metals in Soil and its Application in Iraq. Global Journal of Engineering science and Researches. 2016; 3, 3, 75-81.

3. LNM Tawfiq and AK Jabber. Steady State Radial Flow in Anisotropic and Homogenous in Confined Aquifers. Journal of Physics: Conference Series. 2018; 1003, 1, 1-12.

4. G Adomian. A review of the decomposition method in applied mathematics. J. Math. Anal. Appl. 1988; 135, 501-544.

5. Y Cherruault. Convergence of Adomian's method. Math. Comput. Model. 1990; 14, 83-86.

6. Y Cherruault, G Adomianb, K Abbaoui and R Rach. Further remarks on convergence of decomposition method. International Journal of Bio-Medical Computing. 1995; 38, 89-93. 
7. M Chrysos, F Sanchez and Y Cherruault. Improvement of convergence of Adomian's method using Pade approximants. Kybernetes. 2002; 31, 884-895.

8. A Wazwaz and A Gorguis. Exact solutions for heat-like and wave-like equations with variable coefficients. Appl. Math. Comput. 2004, 149, 15-29.

9. A Soufyane and $M$ Boulmalf. Solution of linear and nonlinear parabolic equations by the decomposition method. Appl. Math. Comput. 2015; 162, 687-693.

10. T Achouri and K Omrani. Numerical solutions for the damped generalized regularized long-wave equation with a variable coefficient by Adomian Decomposition method. Commun. Nonlinear. Sci. Numer. Simulat. 2009; 14, 2025-2033.

11. LNM Tawfiq and HW Rasheed. On Solution of Non Linear Singular Boundary Value Problem. IHJPAS. 2013; $26,3,320-328$.

12. LNM Tawfiq and MA Hassan. Estimate the Effect of Rainwaters in Contaminated Soil by Using Simulink Technique. Journal of Physics: Conference Series. 2018; 1003, 1, 1-7.

13. AK Jabber and LNM Tawfiq. New Transform Fundamental Properties and its Applications. Ibn Alhaitham Journal for Pure and Applied Science. 2018; 31, 1,151-163, doi: http://dx.doi.org/10.30526/31.2.1954.

14. LNM Tawfiq, KA Jasim and EO Abdulhmeed. Pollution of soils by heavy metals in East Baghdad in Iraq. International Journal of Innovative Science, Engineering \& Technology. 2015; 2, 6, 181-187.

15. MO Enadi and LNM Tawfiq. New Approach for Solving Three Dimensional Space Partial Differential Equation. Baghdad Science Journal. 2019; 16, 3, 786-792.

16. H Salih, LNM Tawfiq, ZRI Yahya and SM Zin. Solving Modified Regularized Long Wave Equation Using Collocation Method. Journal of Physics: Conference Series. 2018; 1003, 1, 1-10. Doi :10.1088/17426596/1003/1/012062.

17. MH Ali, LNM Tawfiq and AA Thirthar. Designing Coupled Feed Forward Neural Network to Solve Fourth Order Singular Boundary Value Problem. Revista Aus. 2019; 26, 2, 140-146. DOI: 10.4206/aus.2019.n26.2.20. 